\title{
German-Language Version of the Compensatory Health Belief Scale
}

\section{Assessing Its Psychometric Properties}

\author{
Theda Radtke ${ }^{1}$, Urte Scholz ${ }^{1}$, Roger Keller ${ }^{2}$, Sonja Perren ${ }^{3}$, and Rainer Hornung ${ }^{4}$ \\ ${ }^{1}$ Department of Developmental and Health Psychology, University of Konstanz, Konstanz, Germany \\ ${ }^{2}$ Zurich University of Teacher Education, Zurich, Switzerland \\ ${ }^{3}$ University of Teacher Education Thurgau, Kreuzlingen, Switzerland \\ ${ }^{4}$ Department of Social and Health Psychology, University of Zurich, Zurich, Switzerland
}

\begin{abstract}
This study constitutes the first psychometric examination of the German-language version of the Compensatory Health Belief (CHB) scale to assess the belief that unhealthy behavior can be compensated for by engaging in healthy behavior. Data from four different Swiss samples of 1,571 adolescents/students (age range 1555 years), collected between 2007 and 2009, were used. Confirmatory factor analysis did not support either the hypothesized 4-factor structure or a second-order factor structure with a latent overall variable for the German-language CHB scale. These results support the inconsistent patterns of CHBs found across European cultures. Thus, the development of behavior-specific scales might be of advantage. Further recommendations for an improvement of the measurement of compensatory health beliefs are discussed.

Key words: Compensatory Health Beliefs scale, German-language version, psychometric properties, Switzerland, cognitive dissonance
\end{abstract}

Psychometrische Überprüfung der deutschen Skala zur Messung kompensatorischer Gesundheitsüberzeugungen

Zusammenfassung. Kompensatorische Gesundheitsüberzeugungen (CHB) werden definiert als der Glaube, dass man negative Gesundheitsfolgen einer ungesunden Verhaltensweise durch das Ausführen eines gesunden Verhaltens kompensieren kann. Diese Studie überprüfte erstmalig die psychometrischen Eigenschaften der deutschen Skala zur Messung kompensatorischer Gesundheitsüberzeugungen anhand von vier unterschiedlichen in der Schweiz zwischen 2007 und 2009 erhobenen Stichproben. Insgesamt nahmen 1571 Jugendliche bzw. Schüler/innen (15 55 Jahre) teil. Konfirmatorische Faktorenanalysen konnten sowohl für die angenommene VierFaktoren-Struktur als auch für einen übergeordneten Faktor zweiter Ordnung keine Bestätigung finden. Diese Ergebnisse stützen die inkonsistenten Befunde bezüglich der CHB Faktor-Struktur in verschiedenen europäischen Ländern. Aufgrund der Ergebnisse wird empfohlen, statt einer generellen CHB Skala verhaltensspezifische Skalen zu entwickeln. Darüber hinaus werden weitere Empfehlungen für eine Verbesserung der Messung der kompensatorischen Gesundheitsüberzeugungen diskutiert.

Schlüsselwörter: kompensatorische Gesundheitsüberzeugungen, deutsche Skala, psychometrische Überprüfung, Schweiz, kognitive Dissonanz

\section{Theoretical background}

A large number of epidemiological surveys have demonstrated that behavior which is regarded as unhealthy is widespread. For example, survey data published by the American Cancer Society indicate that there are almost 1 billion male and 250 million female smokers worldwide (Shafey, Eriksen, Ross, \& Mackay, 2009). In addition, the World Health Organization (WHO) estimates that about 2

Work by the authors was partly supported by the Tabakpräventionsfonds (Swiss Tobacco Prevention Fund), and the Stiftung Suzanne und Hans Biäsch zur Förderung der Angewandten Psychologie (Suzanne and Hans Biäsch Foundation to Support Applied Psychology). The authors would like to thank all of their student assistants, especially Vesna Belan. billion people worldwide consume alcoholic beverages (excluding individuals with diagnosable alcohol use disorders) and that approximately $60 \%$ of the world's population do not reach the recommended amount of physical activity required to attain health benefits (WHO, 2004a, 2004b).

These data demonstrate very clearly that a majority of people worldwide hold patterns of unhealthy behaviors which are the leading causes of diseases and death (McGinnis \& Foege, 1993). Equally, it has commonly been shown that people are concerned about their health, are aware of health risks, and acknowledge that quitting an unhealthy behavior or enhancing a healthy behavior would reduce their risk for diseases (e.g., Colavito, Guthrie, 
Hertzler, \& Webb, 1996; Halpern \& Warner, 1993; Scheier \& Botvin, 1997). This inconsistency in awareness and behavior is a relevant concern, because the contemplation of harmful effects of unhealthy behavior as well as the benefits of reducing an unhealthy behavior and enhancing a health behavior are a central focus of various health campaigns (e.g., health warnings on cigarette packs; Peterson \& Lieder, 2006). Therefore, a better understanding of this discordance could inform the improvement of health promotion campaigns and identify possible barriers in health behavior changes.

In this regard, the question arises of why people can be aware of the substantial harms of unhealthy behaviors and yet stick to the unhealthy but mostly pleasurable behavior. Much research has focused on the theory of cognitive dissonance to explain how people cope with the awareness of being at risk and the guilty conscience toward their health on the one side and the desire to engage in an unhealthy behavior and maximize pleasure on the other side (such as eating a piece of cake and maximizing pleasure in doing so (Festinger, 1957)). Festinger (1957) theorized that people need to relieve this dissonance, which emerges when a person acts in a way that is contrary to their own aims, knowledge, or attitudes. Hence, they resort to various coping methods such as attempts to resist the desire or, alternatively, reevaluation of the harmfulness of the behavior (cf. Bowler, Bowler, \& James, 2011; Festinger, 1957; Trope $\&$ Fishbach, 2000). Another coping strategy to overcome that cognitive dissonance is proposed by the compensatory health belief model referred to as compensatory health beliefs (CHBs; Rabiau, Knäuper, \& Miquelon, 2006).

\section{Compensatory Health Beliefs}

CHBs are thoughts, convictions, and opinions that an unhealthy behavior (e.g., tobacco consumption) can be compensated for (or neutralized) through the performance of a healthy behavior (e.g., eating fruit). According to Rabiau et al. (2006), CHBs can be accurate, partly accurate, or inaccurate. The classification can be difficult, because often unhealthy behavior causes several negative effects on health, and the compensatory behavior only compensates for one adverse effect (e.g., Sinner, Folsom, Harnack, Eberly, \& Schmitz, 2006).

CHBs are automatically activated when a person fails to resist temptations such as drinking alcohol; it is assumed that CHBs reduce cognitive dissonance by justifying unhealthy behavior choices with the intention to engage in some other healthy behavior. This does not mean, however, that people actually carry out the intended compensatory behavior (e.g., exercise the next morning), because the initial dissonance may weaken over time, causing the need to compensate for the unhealthy behavior to fade. In this way, the giving up of unhealthy behaviors is hindered by the CHBs, which actually facilitate the further engaging in unhealthy behaviors without the person feeling guilty about their negative health effects.

Knäuper, Rabiau, Cohen, and Patriciu (2004) developed a scale to assess CHBs. First, they generated an initial item pool by collecting CHBs through an international Internet survey. Respondents were mainly North Americans (49.4\%) or Europeans (36.6\%). In a second stage, Knäuper et al. (2004) reviewed all 523 suggested CHBs according to the CHB definition and deleted all suggestions that did not fit with the concept, as well as all redundant, unclear, and not well worded items. The remaining 40 items were then investigated in a Canadian sample of 381 undergraduate students (mean age 20.9) by means of principal axis factor analysis, retest reliability, and confirmatory factor analysis. The final Canadian CHB scale consists of 17 items (for the English items, see Knäuper et al., 2004) divided into four subscales labeled substance use (Cronbach's $\alpha=.74$ ), eating/sleeping habits $(\alpha=.66)$, stress $(\alpha=.63)$, and weight regulation $(\alpha=.57)$. Cronbach's alpha for the overall scale is .80 and test-retest reliability after a duration of 4.5 months is $r_{t t}=.75$. The four subscales are correlated with each other, but no cross loadings between the items have been found. The answering format was a 5-point Liker-scale ranging from 1 (strongly disagree) to 5 (strongly agree). Next to the reliability, Knäuper et al. (2004) also found evidence for the criterion-related validity of the CHB scale by showing that $\mathrm{CHBs}$ are related to risk behaviors (like smoking).

For a Dutch version (translated by a back-translation procedure) of the overall CHB scale, the internal consistency (Cronbach's $\alpha=.78$ ) and the test-retest reliability $\left(r_{t t}=.82\right)$ have also been proven (de Nooijer, PuijkHekman, \& van Assema, 2009). Furthermore, the factor structure of the Dutch CHB scale was examined by a principal component analysis in a sample of 244 students (de Nooijer et al., 2009). In this Dutch study and in a study conducted by Kaklamanou and Armitage (2012) to investigate CHBs in the United Kingdom, the internal consistency of the subscales was slightly lower, and the factor structure of the Canadian CHB scale could not be replicated. In both studies, no cross loadings between the items resulted.

Until now, there has been little research on CHBs (e.g., de Nooijer et al., 2009; Kaklamanou \& Armitage, 2012; Kronick \& Knäuper, 2010; Rabiau et al., 2006). The present study aims at contributing to the investigation of $\mathrm{CHBs}$, as holding CHBs might preclude individuals from acquiring healthier lifestyles. Findings could additionally have important applications - for example, in health campaigns. For the examination of such processes in German-speaking regions such as Austria, Germany, or 
Switzerland, a first aim is to investigate the psychometric properties of a German translation of the CHB scale (Lippke, Hohmann, Kalusche, \& Knäuper, 2007), which has not yet been investigated thoroughly. The translation of the Canadian CHB scale into German was carried out by Lippke et al. (2007) with the help of the original author of the Canadian CHB scale, who is a native speaker of German. Only small cultural adaptations to the German context were conducted (Lippke et al., 2007).

\section{Research Aim}

Due to the lack of a proper examination of the Germanlanguage CHB scale, the aim of this study was to evaluate empirically the factor structure of the German-language version of the CHB scale (see Table 1 for the German items). First, tests of the internal consistency and the test-retest reliability were conducted. Second, the hypothesized 4-factor structure was assessed. Furthermore, a second-order factor model was defined to test whether the

Table 1. Internal consistency of the 17-item CHB scale and its four subscales in four samples

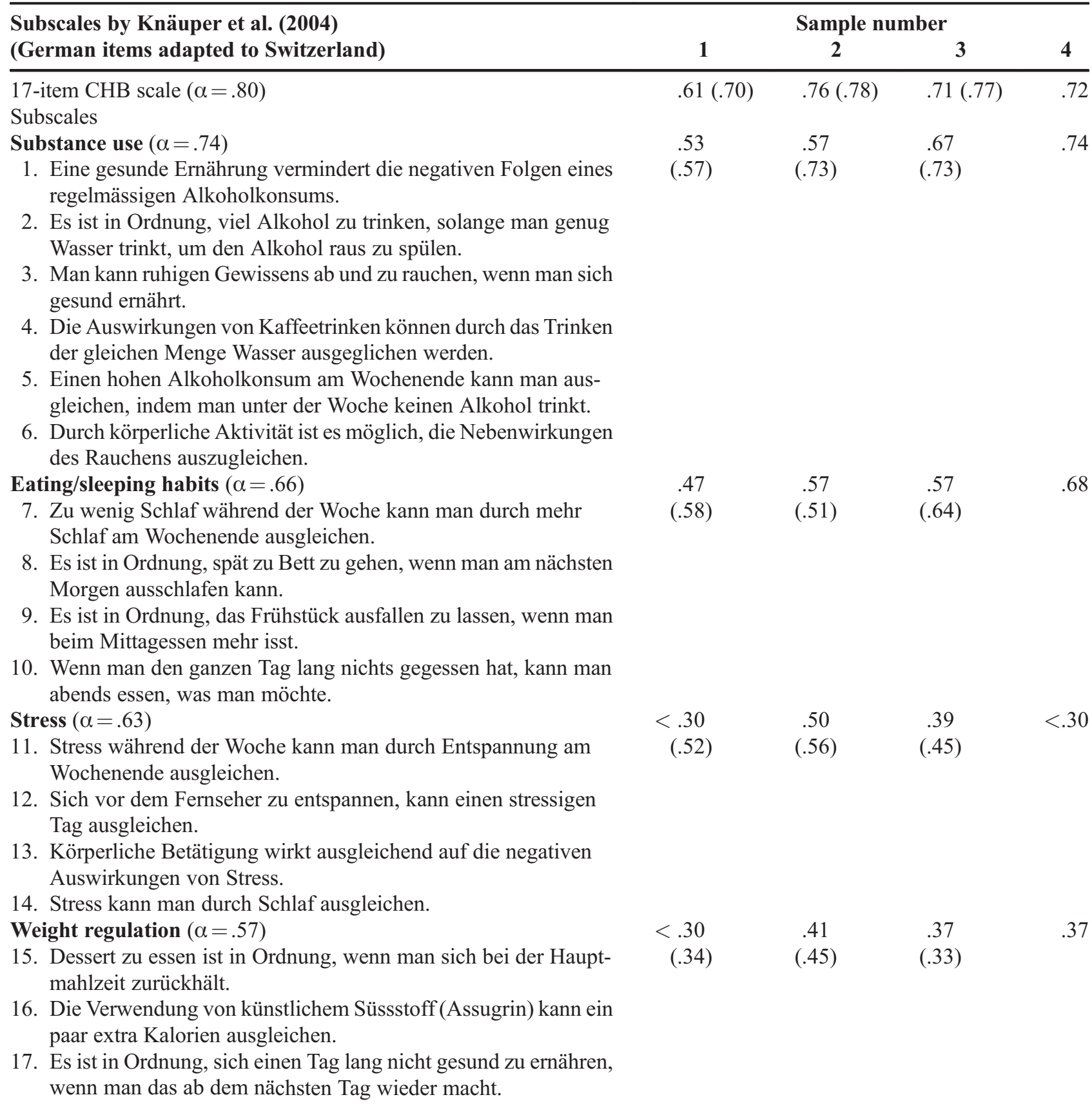

Note: Results for Time 2 of measurement are provided in parentheses. The total sample was used for these analyses. Standard $z$-scores were used in the analyses. CHB compensatory health belief. 
subscales load on an overall CHB scale which is consistent with Knäuper et al. (2004).

\section{Methods}

The present study investigated the CHB scale's factor structure in four different samples and compared their results.

\section{Participants and Procedures}

\section{Sample 1}

Participants were 789 adolescents between 15 and 21 years old, recruited from nine schools in four Germanspeaking cantons in Switzerland to investigate smokingspecific CHBs. Cases with inconsistent or ambiguous data were excluded $(n=64)$. The final sample included 725 participants ( 455 girls, 270 boys) from a total of 56 classes. The average participant was 17.0 years old $(S D=1.35)$. The school levels were the following: upper secondary school $(31.7 \%)$, secondary school $(11.7 \%)$, and vocational school (56.7 \%). Four schools also agreed that their students would complete the paper-and-pencil questionnaire a second time 4 months later $(N=384,15$ - to 21 year-old adolescents with reasonable answers, $n=262$ ). The first survey took place between January and February 2008, and the second survey between May and July 2008. Participants were given no reward.

\section{Sample 2}

For data collection, a computer-assisted telephone interview (CATI)-recruited Internet panel in the Germanspeaking regions of Switzerland was used. Sampling, data collection, and data preparation were performed by the LINK Institute, an independent social research agency. Furthermore, a longitudinal design was chosen with two repeated measurements 4 months apart (between January and October 2009). A total of 480 persons aged 15 to 20 years filled out the online questionnaire at Point of Measurement 1. Cases with ambiguous data were excluded $(n=17)$. The final sample consisted of 463 participants (235 male, 228 female) with a mean age of 17.72 years $(S D=1.66)$. Of the 463 adolescents, 275 participated in the retest. Participants were rewarded with a voucher (for mobile phone credit or for a supermarket) worth CHF 30 (USD 28).

\section{Sample 3}

Participants were 265 first-year German-speaking students (196 women, 69 men) from the University of Zurich,
Switzerland (mean age $=21.3$ years; $S D=5.1$, range $18-$ 55 years) participating in a larger longitudinal online study with nine points of measurement (Perren, Keller, Passardi, \& Scholz, 2010; Scholz, Keller, \& Perren, 2009). For current analyses, only data at Points of Measurement 1 (August 2007) and 9 (taken 4 months later) were included, because CHBs were collected only twice. Of this sample, 252 also participated at Point of Measurement 9. Participants received a voucher for books worth CHF 60 (USD 62) for answering the online questionnaire.

\section{Sample 4}

A total of 118 German-speaking, female undergraduate students from the University of Zurich, Switzerland, studying psychology, participated in a larger longitudinal study at three points of measurement from January to June 2007. For current analyses, only data at Point of Measurement 1 were included, because CHBs were collected only once. The mean age of the female participants was 22.8 years $(S D=5.4$, range $18-43$ years). For completing the paper-and-pencil questionnaire, all individuals participated in a lottery to win one of three vouchers for books worth CHF 30 (USD 31).

In all four samples, participation in the study involved providing anonymous and confidential responses to the questionnaire. Informed consent was obtained from the participants, and they were treated according to the principles of the Declaration of Helsinki (World Medical Association, 2011).

\section{Measures}

The presented samples used the German translation of the 17-item CHB scale (Lippke et al., 2007) described above (see Table 1). Small adaptations were implemented, such as the adjustment of notation to the Swiss spelling, or the substitution of a few words to fit the Swiss expression (e.g., the Swiss use different wordings for desserts/sweets than Germans do). In Samples 1 and 2, the CHBs were measured using a 5-point Likert scale, ranging from 1 (strongly disagree) to 5 (strongly agree). Sample 3 used a 7-point Likert scale of 1 (strongly disagree) to 7 (strongly agree), and in Sample 4, CHBs were measured using a 7point Likert scale from 0 (strongly disagree) to 6 (strongly agree). For all factor analyses, standard $z$-scores were used.

\section{Data Analysis}

Descriptive statistics were assessed using SPSS 15.0: for example, the internal consistency was calculated using Cronbach's alpha, and missing values were replaced by the expectation maximization (EM) imputation method (cf. 
Table 2. Scale characteristics (mean, $S D$, range, and skewness)

\begin{tabular}{lcccc}
\hline Sample number & $\boldsymbol{M}$ & $\boldsymbol{S D}$ & Range & Skewness \\
\hline $1(N=725)$ & 2.79 & .39 & $1-5$ & -.15 \\
$2(N=463)$ & 2.81 & .51 & $1-5$ & .18 \\
$3(N=265)$ & 3.04 & .66 & $1-7$ & .19 \\
$4(N=118)$ & 2.72 & .74 & $0-6$ & .33 \\
\hline
\end{tabular}

Tabachnick \& Fidell, 2007). Overall, the number of missing data points caused by item nonresponse was very low at $<5 \%$. Due to dropouts from Measurement 1 to Measurement 2, within Sample 1 (61.6\%) and 2 (40.6\%), the number of missing values was higher, but dropout analyses revealed no significant difference between the dropout and the original sample in relation to the $\mathrm{CHBs}$ and sociodemographic factors. Confirmatory factor analyses (CFAs) were performed by Mplus 6.12 (Muthén \& Muthén, 2007). The structural analyses were based on the a priori model of a 4-factor structure found by Knäuper et al. (2004). In addition to the analysis of the first-order factor structure of four hypothesized factors and in accordance with Knäuper et al. (2004), the second-order factor structure was also examined with an overall CHB scale as the latent variable. The CFA goodness-of-fit indices are presented: In addition to the chi-square statistic, the comparative fit index (CFI), the Tucker-Lewis index (TLI), the root mean square error of approximation (RMSEA), and the standardized root mean square residual (SRMR) were utilized. An acceptable model fit is indicated by a CFI and TLI value greater than .90 . Furthermore, for the RMSEA and the SRMR, a cutoff value of .05 or lower represents good model fit (Hu \& Bentler, 1999). The data structure of Sample 1 was hierarchical, because participants were nested within school classes. Consequently, multilevel modeling was used within the CFA to correct the standard errors and the chi-square test of model fit due to nonindependence of the observations.

When the CHB scale was completed at two points of measurement, test-retest reliability was calculated using Pearson correlations, and the CFA was repeated with the retest sample.

\section{Results}

\section{Descriptive Statistics}

The main descriptive aspects of the CHB scale are presented in Table 2 for each sample.

\section{Reliability}

The reliability analyses for the subscales and the overall scale were carried out for each sample to enable a comparison with the Canadian and Dutch CHB scales (see Table 1). Overall, it can be stated that the reliability of each subscale could only marginally be improved by dropping items.

\section{Internal Consistency}

The alpha coefficient for the overall scale ranged from $\alpha=.61$ to .76 . The internal consistency for the subscales resulted in lower Cronbach's alpha values compared with the original Canadian scale. Especially for the subscales stress and weight regulation, Cronbach's alpha values were extremely low.

\section{Test-Retest Reliability}

Test-retest coefficients were comparable across Sample 3, $r_{t t}=.64(p \leq .01, n=252)$, Sample $1, r_{t t}=.60(p \leq .001$, $n=262)$, and Sample 2, $r_{t t}=.60(p \leq .01, n=275)$.

\section{Confirmatory Factor Analysis}

\section{First-Order Factor Analysis}

CFA was conducted to confirm the factor structure of the original Canadian scale. A model was tested with four correlated factors as latent variables. In Table 1, the number of specified items can be seen for each factor. The factor loading of the first item of each of the four factors was set to 1 . All remaining items were allowed to vary freely. Residuals were not allowed to correlate (Muthén \& Muthén, 2007). In all subsequent CFAs, the maximum likelihood estimator was used.

Overall, the fit indices failed to provide an adequate fit of the data in each of the four samples (see Table 3). For the model described above, the chi-square value indicated that the model did not fit the data well. Furthermore, there was a rather low CFI and TLI value. The RMSEA, and to the same extent the SRMR, also indicated an unacceptable fit. Likewise, not all of the factor loadings were significant. For some items a low $R^{2}(<.200)$ was found in each sample, which suggests that the items may not really be reflecting the same content (e.g., Item 7, 9, or 14; cf. Table 1). The remaining items had $R^{2}$ values that ranged 
Table 3. Fit indices for the 17-item CHB scale: Test of 4-factor structure and second-order factor structure (in parentheses)

\begin{tabular}{lcccc}
\hline Index & $\mathbf{1}$ & $\mathbf{2}$ & $\mathbf{3}$ & $\mathbf{4}$ \\
\hline$N$ & 725 & 463 & 265 & 118 \\
$\chi^{2}$ & $319.93(325.66)$ & $302.18(308.55)$ & $269.55(277.57)$ & $142.07(147.88)$ \\
$\chi^{2} \mathrm{df}$ & $113(115)$ & $113(115)$ & $113(115)$ & $113(116)$ \\
$p$ & $.00(.00)$ & $.00(.00)$ & $.00(.00)$ & $.03(.02)$ \\
CFI & $.73(.73)$ & $.79(.79)$ & $.74(.73)$ & $.91(.90)$ \\
TLI & $.68(.68)$ & $.06(.06)$ & $.68(.68)$ & $.89(.88)$ \\
RMSEA & $.05(.05)$ & $.06(.06)$ & $.07(.07)$ & $.05(.05)$ \\
SRMR & $.05(.06)$ & $.08(.08)$ & $.08(.07)$ \\
\hline
\end{tabular}

Note: Confirmatory factor analysis (CFA) was performed with multilevel CFA for Sample 1. The number of items for each factor can be seen in Table 1. Standard $z$-scores were used in the analyses. CHB compensatory health belief; CFI comparative fit index; TLI Tucker-Lewis index; RMSEA root mean square error for approximation; SRMR standardized root mean square residual.

from .20 to .76 , but the majority of the $R^{2}$ values were rather low. Moreover, the communality was low for most items (scale characteristics and $h^{2}$ values of each item are available upon request).

Consistently, the fit indices for the retest samples were as follows: Retest Sample 1, $\chi^{2}(113, N=262)=253.60$; $p \leq .001 ; \mathrm{CFI}=.69 ; \mathrm{TLI}=.63 ; \mathrm{RMSEA}=.07$; retest Sample 2, $\chi^{2} \quad(113, \quad N=275)=246.32 ; \quad p \leq .001$; $\mathrm{CFI}=.83 ; \mathrm{TLI}=.79 ; \mathrm{RMSEA}=.07$; and retest Sample $3, \chi^{2}(113, N=252)=304.83 ; p \leq .001 ; \quad \mathrm{CFI}=.74$; $\mathrm{TLI}=.69 ; \mathrm{RMSEA}=.08$.

\section{Second-Order Factor Analysis}

In addition to the confirmatory first-order factor analysis, a second-order factor structure was examined with an overall $\mathrm{CHB}$ scale as the latent variable. As shown in Table 3, the goodness-of-fit indices were almost identical with those of the first-order factor analysis.

\section{Discussion}

This paper compared the German-language version's factor structure of the $\mathrm{CHB}$ scale in four samples to the one found by Knäuper et al. (2004). Neither did the confirmatory factor analyses confirm the existence of a 4factor structure, nor could a second-order factor structure with a latent overall variable be found for the Germanlanguage $\mathrm{CHB}$ scale. In addition, internal consistency for the CHB subscales was unsatisfactory. The results were unsurprising, in light of the findings of de Nooijer et al. (2009) and Kaklamanou and Armitage (2012). Both studies also could not replicate the factor structure of the Canadian CHB scale and also yielded lower Cronbach's alpha values for the subscales than those of the original CHB scale. Indeed, Kaklamanou and Armitage (2012) identified only three factors instead of four, which were labeled as sleep pattern, stress, and exercise. One possible key explanation for these inconsistent patterns across different cultures may be that European cultures hold other compensatory health beliefs than people in Canada. In view of the diverse health-related problems and different resulting health policies, it is rather likely that people from different countries hold different CHBs (WHO, 2004a, 2004b). Considering this, one challenge for future research might be the generation of country-specific CHB item pools, especially because the item pool in Canada was generated on an empirical but not a theoretical basis.

A further explanation for the unconfirmed subscale structure of the original $\mathrm{CHB}$ scale might be that participants' agreement or disagreement with some CHB items is not clearly interpretable, due to the if-structure of six items (e.g., "Smoking from time to time is ok if one eats healthy food"). Therefore, it is possible that participants responded only to the first part of the item, rather than to the CHB item as a whole. This might produce inconsistent patterns within the factor structure.

Furthermore, irregular time periods were used in the 17 items. For example, Items 7 and 11 included compensating behavior over the weekend, Item 5 during the week, and Items $8,10,12$, and 17 on the same day or the next day, whereas all other items contained no time indication. It can be supposed that everyday habits (e. g., eating or sleeping) and activities (e.g., exercise) differ across populations. For example, Oltersdorf, Schlettwein-Gsell, and Winkler (1999) stated that people from different cultures have diverse eating patterns and that the temporal structure of food and drink intake also varies. They mentioned that a cooked meal at lunch time is the rule in Switzerland, whereas it is eaten at dinnertime in the United Kingdom or the United States. The National Sleep Foundation (NSF, 2010) also pointed out that sleeping habits are quite different among varying ethnic groups, such as Whites, African Americans, or Asians. Furthermore, the NSF revealed that these ethnic groups differ in the reported 
amount of required sleep each night to perform best during the next day, which might impact compensatory beliefs. In sum, it is to be assumed that the expectations about feasible compensating behaviors and the importance of different time spans varied in populations/ethnic groups and might influence the constitution of CHBs. Therefore, it is proposed that future studies should investigate $\mathrm{CHBs}$ in more detail for the German-speaking Swiss context, including which compensatory beliefs are activated by which unhealthy behavior. The role of time periods should also be taken into account. In line with Radtke, Scholz, Keller, Knäuper, and Hornung (2011), we further suggest developing behavior-specific CHB scales, each for one specific behavior (e.g., eating and sleeping habits) that needs to be compensated for, rather than for many different unhealthy behaviors combined. Thus, it would be possible to enhance internal consistency of the subscales and to account for more compensating strategies for each behavior. Furthermore, we assume that by use of behavior-specific scales, the concept of CHBs becomes more understandable for the respondents. Radtke, Scholz, Keller, and Hornung (2012) already demonstrated that a behavior-specific CHB scale is predictive for the intention to stop a specific unhealthy behavior.

When developing such scales, the findings of Kaklamanou, Armitage, and Jones (2013) should also be taken into account. These authors demonstrated that respondents sometimes have difficulties in distinguishing between the compensatory belief and the compensatory behavior. This might be another reason for why the factor structure could not be replicated - because some people might answer the items with regard to beliefs and others with regard to behaviors. Therefore, future research should make an explicit distinction between the belief and behavior when measuring $\mathrm{CHBs}$.

\section{Limitations}

One possible limitation of this study was that the Germanlanguage version of the CHBs was used. Only small cultural adaptations for the Swiss context were implemented, to ensure comparability with the German context. It can be supposed that some German words such as that for artificial sweeteners are unfamiliar for people speaking Swiss German as their mother tongue. Moreover, the German CHB scale should be verified by forwardbackward translation to avoid inaccuracy within the translation process. Another limitation might be that the German-language version of the CHB scale was only tested in samples of adolescents and young adults. The generalizability of our results for middle-aged or older adults is, therefore, limited. Further research is recommended to examine CHBs in samples with wider age ranges. Thus far, CHBs have not been investigated for older age groups. Evidence that $\mathrm{CHBs}$ might be more relevant in adults is provided by Xu's (2002) study of smokers. He pointed out that adult smokers, with their longer smoking history, might be more concerned about their health, and consequently more often feel a cognitive dissonance concerning smoking. For this reason they would have higher values on the CHB scale. In addition, the sample sizes in some of the four samples were expandable. However, the sample sizes were acceptable for all analyses, and the availability of four different samples to examine the $\mathrm{CHB}$ scale is worth mentioning.

\section{Conclusion}

This study is the first to investigate the psychometric properties of the German-language version of the CHB scale. The findings contribute to existing studies concerning CHBs. The factor structure of the CHB scale could not be replicated for the German-language version, which is consistent with other studies carried out in Europe (de Nooijer et al., 2009; Kaklamanou \& Armitage, 2012). Therefore further development of behavior-specific CHB scales such as a diet- or smoking-specific CHB or a diabetes-specific CHB scale about glucose testing (Lippke et al., 2007; Rabiau, Knäuper, Nguyen, Sufrategui, \& Polychronakos, 2009; Radtke et al., 2011, 2012) is recommended. When developing new behavior-specific CHB scales, the limitations of the general CHB scale mentioned above, such as using identical time frames for the items, should be taken into account. Overall, further improvement of the measurement of CHBs is important because it allows a more precise investigation of compensatory health beliefs and their associations with healthbehavior change.

\section{References}

Bowler, J. L., Bowler, M. C., \& James, L. R. (2011). The cognitive underpinnings of addiction. Substance Use and Misuse, 46, 10601071.

Colavito, E. A., Guthrie, J. F., Hertzler, A. A., \& Webb, R. E. (1996). Relationship of diet health attitudes and nutrition knowledge of household meal planners to the fat and fiber intakes of meal planners and preschoolers. Journal of Nutrition Education, 28, 321328.

de Nooijer, J., Puijk Hekman, S., \& van Assema, P. (2009). The compensatory health beliefs scale: Psychometric properties of a cross culturally adapted scale for use in the Netherlands. Health Education Research, 24, 811817.

Festinger, L. A. (1957). A theory of cognitive dissonance. Stanford, CA: Stanford University Press.

Halpern, M. T., \& Warner, K. E. (1993). Motivations for smoking cessation: A comparison of successful quitters and failures. Journal of Substance Abuse, 5, 247256.

Hu, L., \& Bentler, P. M. (1999). Cutoff criteria for fit indices in covariance structure analysis: Conventional criteria versus new alternatives. Structural Equation Modeling, 6, 155. 
Kaklamanou, D., \& Armitage, C. J. (2012). Testing compensa tory health beliefs in a UK population. Psychology and Health, 27, 10621074.

Kaklamanou, D., Armitage, C. J., \& Jones, C. R. (2013). A further look into compensatory health beliefs. British Journal of Health Psychology, 18, 139154.

Knäuper, B., Rabiau, M., Cohen, O., \& Patriciu, N. (2004). Compensatory health beliefs scale development and psy chometric properties. Psychology and Health, 19, 607624 .

Kronick, I., \& Knäuper, B. (2010). Temptations elicit compen satory intentions. Appetite, 54, 398401.

Lippke, S., Hohmann, C., Kalusche, A., \& Knäuper, B. (2007) Compensatory beliefs scale general and diet (German). Retrieved from http://www.psych.mcgill.ca/perpg/fac/ knaeuper/GermanCHB.doc

McGinnis, J. M., \& Foege, W. H. (1993). Actual causes of death in the United States. Journal of American Medical Associ ation, 270, 22072212.

Muthén, L. K., \& Muthén, B. O. (2007). Mplus user's guide (5th ed.). Los Angeles: Muthén \& Muthén.

National Sleep Foundation. (2010). 2010 Sleep in America poll: Summary of findings. Washington, DC: National Sleep Foundation.

Oltersdorf, U., Schlettwein Gsell, D., \& Winkler, G. (1999). Assessing eating patterns: An emerging research topic in nutritional sciences: Introduction to the symposium. Appe tite, 32, 17 .

Perren, S., Keller, R., Passardi, M., \& Scholz, U. (2010). Well being trajectories across transitions: The Development of a retrospective measure. Swiss Journal of Psychology, 69, 15 29.

Peterson, L. E., \& Lieder, F. (2006). Die Effektivität von schriftlichen und graphischen Warnhinweisen auf Zigaret tenschachteln. Eine Überprüfung des revidierten Modells der Schutzmotivation [The effectiveness of written and graphic health warnings on cigarette packs: An examination of the revised model of protection motivation]. Zeitschrift für Sozialpsychologie, 37, 245258.

Rabiau, M., Knäuper, B., \& Miquelon, P. (2006). The external quest for optimal balance between maximizing pleasure and minimizing harm: The compensatory health beliefs model. British Journal of Health Psychology, 11, 139153.

Rabiau, M., Knäuper, B., Nguyen, T. K., Sufrategui, M., \& Polychronakos, C. (2009). Compensatory beliefs about glucose testing are associated with low adherence to treat ment and poor metabolic control in adolescents with type 1 diabetes. Health Education Research, 24, 890896.

Radtke, T., Scholz, U., Keller, R., \& Hornung, R. (2012). Smoking is ok as long as I eat healthily: Compensatory health beliefs and their role for intentions and smoking within the health action process approach. Psychology and Health, 27, 91107.

Radtke, T., Scholz, U., Keller, R., Knäuper, B., \& Hornung, R. (2011). Smoking specific compensatory health beliefs and the readiness to stop smoking in adolescents. British Journal of Health Psychology, 16, 610625.

Scheier, M. L., \& Botvin, G. J. (1997). Expectancies as mediators of the effects of social influences and alcohol knowledge on adolescent alcohol use: A prospective analysis. Psychology of Addictive Behaviors, 11, 4864.

Scholz, U., Keller, R., \& Perren, S. (2009). Predicting behavioral intentions and physical exercise: A test of the Health Action Process Approach at the intrapersonal level. Health Psy chology, 28, 702708.

Shafey, O., Eriksen, M., Ross, H., \& Mackay, J. (2009). The tobacco atlas (3rd ed.). Atlanta, GA: American Cancer Society.

Sinner, P., Folsom, A. R., Harnack, L., Eberly, L. E., \& Schmitz, K. H. (2006). The association of physical activity with lung cancer incidence in a cohort of older women: The Iowa Women's Health Study. Cancer Epidemiology, Biomarkers \& Prevention, 15, 23592363.

Tabachnick, B. G., \& Fidell, L. S. (2007). Using multivariate statistics (5th ed.). Boston: Pearson.

Trope, Y., \& Fishbach, A. (2000). Counteractive self control in overcoming temptation. Journal of Personality and Social Psychology, 79, 493506

World Health Organization. (2004a). Global status report on alcohol 2004. Geneva: Department of Mental Health and Substance Abuse.

World Health Organization. (2004b). Global strategy on diet, physical activity and health. Geneva: Author.

World Medical Association. (2011). WMA Declaration of Helsinki: Ethical principles for medical research involving human subjects. Retrieved from http://www.wma.net/en/ 30publications/10policies/b3/index.html.pdf

$\mathrm{Xu}, \mathrm{K}$. T. (2002). Compensating behaviors, regret, and hetero geneity in the dynamics of smoking behavior. Social Science \& Medicine, 54, 133146.

Dr. Theda Radtke

Department of Psychology

Developmental and Health Psychology

University of Konstanz

Postbox 5560/Box 14

78457 Konstanz

Germany

E Mail: Theda.Radtke@uni konstanz.de 